Peter Topping

\title{
The isoperimetric inequality on a surface
}

Received: 31 July 1998

Abstract. We prove a new isoperimetric inequality which relates the area of a multiply connected curved surface, its Euler characteristic, the length of its boundary, and its Gaussian curvature.

\section{Introduction}

Let $G$ be a domain with compact closure $\bar{G}$ on a complete (possibly compact) orientable Riemannian surface $\mathcal{M}$ with a $C^{2}$ smooth metric. Suppose that the boundary $\partial G$ of $G$ exists, and consists of a finite number of disjoint simple closed piecewise regular curves ${ }^{1}$. We denote the area of $G$ by $A$, the total length of $\partial G$ by $L$, and the Euler characteristic of $G$ by $\chi$. The Gaussian curvature of the surface, denoted $K$, induces the rearrangement $K^{*}:(0, A) \rightarrow \mathbb{R}$ defined to be the unique decreasing function with the property that

$$
\operatorname{Area}(\{x \in G \mid K(x) \geq s\})=\left|\left\{y \in(0, A) \mid K^{*}(y) \geq s\right\}\right|,
$$

for all $s \in \mathbb{R}$. (So $K^{*}$ is the inverse of $g(s):=\operatorname{Area}(\{x \in G \mid K(x) \geq s\}$ ) whenever $g$ is continuous on $\mathbb{R}$.) Our main theorem is then as follows.

Theorem 1. With $G$ as described above, there holds the isoperimetric inequality

$$
4 \pi A \chi \leq L^{2}+2 \int_{0}^{A}(A-x) K^{*}(x) d x .
$$

In a recent article [5] we announced this result in the simply connected case (when $\chi=1$ ), and gave a swift proof, under extra hypotheses, using the curve shortening flow. We also described how it generalises the inequalities of Alexandrov, Fiala-Huber, Bol, Bernstein-Schmidt and others, which we recall in the following corollary.

Corollary 1. With $G$ as in Theorem 1 and $\chi=1$, we have the following classical inequalities:

P. Topping: Department of Mathematics, Cornell University, Ithaca, NY 14853, USA. e-mail: topping@math.cornell.edu

1 "Piecewise regular" in this paper will mean that the curve is described by a continuous curve $\gamma$ which, apart from at finitely many points, is $C^{2}$ regular and satisfies $\gamma^{\prime} \neq 0$. 
(i) (Alexandrov) Given $K_{0} \in \mathbb{R}$,

$$
4 \pi A \leq L^{2}+K_{0} A^{2}+2 A \int_{D}\left(K-K_{0}\right)_{+},
$$

(ii) (Fiala-Huber)

$$
4 \pi A \leq L^{2}+2 A \int_{D} K_{+},
$$

(iii) (Bol) If $K \leq K_{0} \in \mathbb{R}$,

$$
4 \pi A \leq L^{2}+K_{0} A^{2}
$$

(iv) (Bernstein-Schmidt) If $K$ is constant,

$$
4 \pi A \leq L^{2}+K A^{2} .
$$

The way in which these inequalities follow from our Theorem 1 was described in [5] and this proof remains valid in our present situation even though we are now considering much more general domains $G$. We repeat only the simplest case of the Bernstein-Schmidt inequality for which $K$ is constant. We then have $K^{*} \equiv K$, and the final term in (1) may simply be integrated to yield $4 \pi A \leq L^{2}+K A^{2}$.

Theorem 1 is sharp in the sense that equality is achieved in (1) for the disc equipped with any rotationally symmetric metric such that the Gauss curvature is nonincreasing as we move radially towards the boundary.

The proof we shall offer uses the method of boundary equidistants (also known as interior parallels) on polyhedral approximations of $G$. The use of polyhedral approximations in the context of proving isoperimetric inequalities seems to go back to the last century, but has been greatly developed since by Alexandrov, Burago and Zalgaller and others (see [1] and [2]). Having chosen this route, we draw heavily on these developments and adopt, where possible, the notation of [2] for ease of reference. The proof we get using this method is somewhat less elegant than that which we gave in [5], but it has the indisputable advantage of requiring no boundary convexity or total curvature constraint.

We shall not attempt to prove the result on polyhedra (since there are problems with the notion of $K^{*}$ ); instead we get an intermediate result on polyhedra and get the full result by passing to the limit of polyhedral approximations. An alternative method which would avoid this peculiarity would be to approximate the metric by a real analytic metric (using, for example, the Gauss curvature flow on $\mathcal{M}$ ) and approximate the boundary by real analytic curves (using, for example, the curve shortening flow) and then bring into play the machinery of Fiala [3] who analysed the structure of the boundary equidistants in the real analytic case.

We should remark that the hypotheses we make on the regularity of the metric on $\mathcal{M}$ and the boundary of $G$ may be easily weakened, though at the expense of clarity of the proof.

Following the completion of this work, Frank Morgan informed us that a similar result to our Theorem 1 has just been obtained independently by Morgan-HutchingsHowards and is to be found in [4]. 


\section{An equivalent formulation of Theorem 1}

Let us recall some properties of the rearrangement $K^{*}$. To begin with, $K^{*}$ has the same image as $K$, and in particular it is bounded from above and below in our situation. The main property of decreasing rearrangements is that they may be used to control integrals of the original function. In particular we have

$$
\int_{G} K=\int_{0}^{A} K^{*},
$$

or more generally, for any $s \in \mathbb{R}$, there holds the equality

$$
\int_{\Sigma_{s}} K=\int_{0}^{\operatorname{Area}\left(\Sigma_{s}\right)} K^{*},
$$

where $\Sigma_{s}=\{x \in G \mid K(x) \geq s\}$. Given a more general measurable set $\Sigma \subset G$ we have the related estimates

$$
\int_{\Sigma} K \leq \int_{0}^{\operatorname{Area}(\Sigma)} K^{*}
$$

and

$$
\int_{\Sigma} K \geq \int_{A-\operatorname{Area}(\Sigma)}^{A} K^{*} .
$$

We now need the notion of "rotation" of a simple closed regular curve $\gamma$ in $G$ which we define to be

$$
\tau(\gamma):=\int_{\gamma} \kappa,
$$

where $\kappa$ is the curvature of $\gamma$ with the sign chosen to be positive as $\gamma$ curves to what we will shall call the "left" side. If we allow $\gamma$ to be merely a simple closed piecewise regular curve, and we denote by $\alpha_{i}$ the angles formed on the left side of $\gamma$ at the points where $\gamma^{\prime}$ is discontinuous, then the definition of $\tau$ may be generalised to

$$
\tau(\gamma)=\int_{\gamma} \kappa+\sum_{i}\left(\pi-\alpha_{i}\right) .
$$

In this way, if $\Sigma$ is a domain whose boundary is described by a collection of curves $\gamma$ (which keep $\Sigma$ on the left side) then we have the Gauss-Bonnet formula

$$
\tau(\partial \Sigma):=\tau(\gamma)=2 \pi \chi(\Sigma)-\int_{\Sigma} K .
$$

By combining (2) and (5) with $\Sigma=G$, we see that

$$
4 \pi A \chi=2 A \tau(\partial G)+2 A \int_{0}^{A} K^{*},
$$

which gives us the following theorem, equivalent to Theorem 1. 
Theorem 2. With $G$ as in Theorem 1, we have the inequality

$$
2 A \tau(\partial G) \leq L^{2}-2 \int_{0}^{A} x K^{*}(x) d x .
$$

We will prove Theorem 1 by proving Theorem 2. This latter formulation also has applications to the theory of geodesics.

Corollary 2. Suppose that $G$ is as in Theorem 1, but with the additional hypothesis that the boundary of $G$ is made up of geodesics in $\mathcal{M}$. Then

$$
L^{2} \geq \int_{0}^{A} x K^{*}(x) d x
$$

\section{Preliminaries and a heuristic description of the proof}

In this section we introduce some notation concerning boundary equidistants, and under assumptions concerning their structure (which are simply false in general) we give a quick proof of Theorem 1 which will convey the idea of the proof in the general case. The use of polyhedral approximations in this work is motivated purely by the fact that they simplify the analysis of the boundary equidistants.

Let $\rho: G \times G \rightarrow \mathbb{R}$ be the metric, in the sense of a metric space, which is induced from the surface $\mathcal{M}$ (i.e. $\rho(x, y)$ is the infimum of the lengths of the curves in $\mathcal{M}$ joining $x$ and $y$ ). The boundary equidistants are defined to be the sets

$$
l_{t}=\{x \in G \mid \rho(x, \partial G)=t\}
$$

which are nonempty precisely for $t \in[0, r]$ where

$$
r=\sup \{\rho(x, \partial G) \mid x \in G\} .
$$

We denote the length of $l_{t}$ by $l(t)$, and remark that as one would expect, the set $l_{t}$ has zero area for all $t \in[0, r]$. (This fact follows from a local analysis coupled with a simple covering argument.) We must also define the sets

$$
P_{t}=\{x \in G \mid \rho(x, \partial G)<t\},
$$

and

$$
G_{t}=\{x \in G \mid \rho(x, \partial G)>t\},
$$

and we denote the area of $P_{t}$ by $f(t)$. Since $l_{r}$ has no area, these definitions imply that $f(r)=A$. In general, we denote the length of a set $\sigma$ by $L(\sigma)$ and the area of a set $\Sigma$ by $A(\Sigma)$. We will have cause to consider various integrals of the Gaussian curvature $K$ over a set $\Sigma$, and in preparation we define

$$
\omega(\Sigma)=\int_{\Sigma} K, \quad \Omega(\Sigma)=\int_{\Sigma}|K| .
$$

In this section only, we will assume that for each $t \in[0, r)$ the set $l_{t}$ is a smooth simple closed curve. This is clearly a severe restriction, but is nevertheless the case 
when $G$ is the 2-disc equipped with a rotationally symmetric metric, and we may construct other domains with this property on any surface using the exponential map.

Given our assumption, standard formulae give us the relations

$$
f^{\prime}(t)=l(t) \geq 0,
$$

and

$$
f^{\prime \prime}(t)=l^{\prime}(t)=-\tau\left(\partial G_{t}\right)=\tau\left(\partial P_{t}\right)-\tau(\partial G)=-\omega\left(P_{t}\right)-\tau(\partial G),
$$

where we have used the fact that $\chi\left(P_{t}\right)=0$ in the Gauss-Bonnet formula. Note that we write $\partial G_{t}$ rather than $l_{t}$ in order to communicate the sign (as described above).

Combining these relations, we find that

$$
\frac{d}{d t}\left(l(t)^{2}\right)=2 f^{\prime}(t)\left(-\omega\left(P_{t}\right)-\tau(\partial G)\right)
$$

Exploiting the property of $K^{*}$ given in (3) then yields

$$
\begin{aligned}
\frac{d}{d t}\left(l(t)^{2}\right)+2 f^{\prime}(t) \tau(\partial G) & \leq-2 f^{\prime}(t) \int_{A-f(t)}^{A} K^{*} \\
& =-2 \frac{d}{d t} \int_{A-f(t)}^{A}(s-A+f(t)) K^{*}(s) d s
\end{aligned}
$$

which we may integrate between $t=0$ and $t=r$ to give

$$
l(r)^{2}-L^{2}+2 A \tau(\partial G) \leq-2 \int_{0}^{A} s K^{*}(s) d s .
$$

Since $l(r)^{2}>0$, we have proved Theorem 2 and therefore Theorem 1 (with the additional hypotheses of this section).

\section{Polyhedral metrics and approximation results}

In order to simplify the study of the structure of the equidistant sets, we will use polyhedral metrics to approximate the metric of $G$. In their book [1], Alexandrov and Zalgaller have developed an extensive theory of such metrics in great generality. Starting from the definition of a polyhedral metric as an "intrinsic" metric (metric in the sense of a metric space) which is everywhere locally isometric to a part of a cone, they are able to define geometric notions such as area and curvature, and establish natural relationships between them. As it turns out, we need not make all these definitions because all of our polyhedral metrics will arise as approximations of the original metric $\rho$ on $G$ in the following way (up to a possible uniform scaling) which automatically induces equivalent geometric notions. 
(i) Pick an appropriate triangulation of $G$ (or at least of a polygon $G^{\prime}$ making up most of $G$ ) where each triangle is bordered by three geodesics in $G^{\prime}$, none of which may be shortened.

(ii) Construct a singular surface by replacing each geodesic triangle by the triangle in the plane with sides of the same length. The sides of each triangle are identified as before.

(iii) Induce the metric of this singular surface onto $G^{\prime}$ using an appropriate 1-1 map between the two surfaces, which is also a bijection between corresponding triangles.

On such a singular surface we clearly have natural notions of lengths of curves and areas of sets, and as we have mentioned above, these are intrinsic quantities (i.e. they may be defined using the metric alone). However, there are also notions of curvature and rotation defined as follows. At any point on the singular surface which is not a "vertex" (i.e. not a point which corresponds to a vertex of the triangulation) the curvature is zero. At a vertex at which the $N$ triangles sharing it make angles $\beta_{1}, \ldots, \beta_{N}$, we define the curvature to be a delta function scaled by a factor $\left(2 \pi-\sum_{1}^{N} \beta_{i}\right)$. By integrating this curvature (or its "absolute value") over a set $\Sigma$, we can give definitions of $\omega(\Sigma)$ and $\Omega(\Sigma)$ analogous to those given earlier.

The rotation of a curve can, in fact, be defined as before (see (4) and (5)). The angles $\alpha_{i}$ are easy to measure at vertices and at edges on a singular surface, though they may also be equivalently defined given just the metric space structure (see [1]). Note that in contrast to the case of rotation on a smooth surface, changing the direction of the curve does not simply change the sign of the rotation if the curve passes through a vertex. Instead, we have the relation

$$
\tau(\gamma)+\tau(-\gamma)=\omega(\gamma)
$$

where $\omega(\gamma)$ is used here to abbreviate $\omega(\operatorname{Image}(\gamma))$.

Using these definitions, we retain the Gauss-Bonnet formula

$$
\tau(\partial \Sigma)=2 \pi \chi(\Sigma)-\omega(\Sigma),
$$

for any domain $\Sigma$ bounded by piecewise regular simple closed curves.

We will make our polyhedral approximations by appealing to the following theorem which was proved in [1] and used in this context in [2].

Theorem 3. Suppose that $G$ is a domain satisfying the conditions of Theorem 1, with metric $\rho$ and associated geometric quantities such as $A, L, \omega, \Omega, \tau$ as defined earlier. Then there exists an increasing sequence of subdomains $G^{i}$ filling out $G$ which are bounded by a finite number of simple closed curves, each a polygon made up of shortest arcs; moreover there exists a sequence of polyhedral metrcs $\rho^{i}$ on $G^{i}$ (with associated geometric quantities $A^{i}, L^{i}, \omega^{i}, \Omega^{i}, \tau^{i}, P_{t}^{i}, G_{t}^{i}, l_{t}^{i}, l^{i}(t)$ and $\left.f_{i}(t)\right)^{2}$ such that

(1) $\rho^{i} \rightarrow \rho$ uniformly, in the sense that for any $\varepsilon>0$ there exists $N \in \mathbb{N}$ so that for any $i \geq N$ and any $x, y \in G^{i}$, we have $\left|\rho(x, y)-\rho^{i}(x, y)\right|<\varepsilon$,

\footnotetext{
${ }^{2}$ For example, we define $\left.P_{t}^{i}=\left\{x \in G^{i} \mid \rho^{i}, \partial G^{i}\right)<t\right\}$.
} 
(2) $\omega^{i}\left(G^{i}\right) \rightarrow \omega(G)$ and $\Omega^{i}\left(G^{i}\right) \rightarrow \Omega(G)$,

(3) $L^{i}:=L^{i}\left(\partial G^{i}\right) \rightarrow L:=L(\partial G)$ and $A^{i}:=A^{i}\left(G^{i}\right) \rightarrow A:=A(G)$,

(4) $A^{i}(Q) \rightarrow A(Q)$ for any $Q \subset \subset G$,

(5) $\omega^{i}(Q) \rightarrow \omega(Q)$ and $\Omega^{i}(Q) \rightarrow \Omega(Q)$ for any $Q \subset \subset G$,

(6) $\tau^{i}:=\tau^{i}\left(\partial G^{i}\right) \rightarrow \tau(\partial G)$,

(7) $\partial G^{i} \rightarrow \partial G$ in the sense that

$$
a_{i}:=\max _{x \in \partial G} \rho\left(x, \partial G^{i}\right) \rightarrow 0, \quad \text { and } \quad b_{i}:=\max _{x \in G \backslash G^{i}} \rho(x, \partial G) \rightarrow 0,
$$

as $i \rightarrow \infty$. Moreover, by adjusting the scaling of the polyhedral metric uniformly, we may assume in addition that

$$
\sup \left\{\rho^{i}\left(x, \partial G^{i}\right) \mid x \in G^{i}\right\}=r:=\sup \{\rho(x, \partial G) \mid x \in G\} .
$$

We now turn to study the boundary equidistants. Let us begin by noting that whilst $\partial G_{t}^{i}$ lies within $l_{t}^{i}$, the two sets need not be equal. This is because a point $x \in l_{t}^{i}$ may be a local maximum for the function $\rho^{i}\left(\cdot, \partial G^{i}\right)$, and is explained in [2] with the aid of pictures.

In [2, Lemma 3.2.2] it was proved that for any $t \in[0, r)$ the boundary $\partial G_{t}^{i}$ consists of a finite number of arcs of curves of constant curvature. For each $i$, the number of such lines is bounded independently of $t \in[0, r)$. Moreover it was proved that there exists a finite number of values $0<t_{1}<t_{2}<\cdots<t_{m}=r$ (depending on $i$ ) such that for $t \in[0, r] \backslash\left\{t_{j}\right\}$, the boundary $\partial G_{t}^{i}$ is equal to the equidistant $l_{t}^{i}$, and consists of a finite number of disjoint simple closed paths (each made up of a finite number of arcs of constant curvature).

After a possible finite largening of the set of values $\left\{t_{j}\right\}$ in the interval $(0, r]$, we have the following control on the equidistants, as proved in [2, Lemma 3.2.3].

Lemma 1. The function $f_{i}$ is continuous on the whole interval $[0, r]$. Moreover, $f_{i}$ is twice differentiable on $[0, r] \backslash\left\{t_{j}\right\}$ with

$$
f_{i}^{\prime}(t)=l^{i}(t) \quad \text { and } \quad f_{i}^{\prime \prime}(t) \leq-\omega^{i}\left(P_{t}^{i}\right)-\tau^{i}\left(\partial G^{i}\right) .
$$

At the singular points $\left\{t_{j}\right\}$ limits from both sides of $f_{i}^{\prime}$ exist, and

$$
f_{i}^{\prime}\left(t_{j}-0\right) \geq f_{i}^{\prime}\left(t_{j}+0\right) .
$$

Note that in [2], $\omega^{i}\left(P_{t}^{i} \cup l_{t}^{i}\right)$ is used in (8) rather than $\omega^{i}\left(P_{t}^{i}\right)$. However, without loss of generality we may assume that the set $\left\{t_{j}\right\}$ is sufficiently large that all the vertices of $G^{i}$ lie in the set $\bigcup_{j} l_{t_{j}}^{i}$, and so for $t \in[0, r] \backslash\left\{t_{j}\right\}$ we may assume that $\omega^{i}\left(P_{t}^{i} \cup l_{t}^{i}\right)=\omega^{i}\left(P_{t}^{i}\right)$.

We will also require a few consequences of Lemma 1 which we collect in a separate lemma.

Lemma 2. There exists a constant $C$ independent of $i$ and $t$ such that $\left|\omega^{i}\left(P_{t}^{i}\right)\right| \leq C$ and $\left|f_{i}(t)\right| \leq C$ and, except for a finite number of values of $t$ (dependent on $i$ ) we have $\left|f_{i}^{\prime}(t)\right| \leq C$. 
Proof. The first part is clear since

$$
\left|\omega^{i}\left(P_{t}^{i}\right)\right| \leq \Omega^{i}\left(P_{t}^{i}\right) \leq \Omega^{i}\left(G^{i}\right) \leq \Omega(G)+1,
$$

for sufficiently large $i$, owing to the convergence of part (2) of Theorem 3 . The second part is as easy, since $f_{i}(t) \in\left[0, A^{i}\right]$ for all $t$ and $A^{i} \leq A+1$ for sufficiently large $i$ by part (3) of Theorem 3. For the final part, we note that owing to the properties of $f_{i}^{\prime}$ given in Lemma 1 , and the fact that $f_{i}^{\prime}(0)=l^{i}(0)=L^{i}\left(\partial G^{i}\right) \leq$ $L+1$ for sufficiently large $i$ (see part (3) of Theorem 3 ) it suffices to bound $f_{i}^{\prime \prime}(t)$ from above, uniformly for $t \in[0, r] \backslash\left\{t_{j}\right\}$. Using Lemma 1 once more, and part (6) of Theorem 3 we find that

$$
f_{i}^{\prime \prime}(t) \leq-\omega^{i}\left(P_{t}^{i}\right)-\tau^{i}\left(\partial G^{i}\right) \leq \Omega(G)+1-\tau(\partial G)+1,
$$

for sufficiently large $i$.

Theorem 3 gives good convergence of curvature integrals over fixed compact sets in $G$ as $i \rightarrow \infty$. However, in practice we shall need convergence of integrals over certain sets depending on $i$.

Lemma 3. For each $t \in[0, r)$, we have the convergence

$$
\omega^{i}\left(P_{t}^{i}\right) \rightarrow \omega\left(P_{t}\right) \quad \text { and } \quad f_{i}(t) \rightarrow f(t),
$$

as $i \rightarrow \infty$.

The key to pinning this lemma down will be the following elementary proposition.

Proposition 1. For any $\varepsilon>0$, there exists $N \in \mathbb{N}$ such that $i>N$ implies that

(1) $G_{t+\varepsilon} \subset G_{t}^{i}$,

(2) $G_{t}^{i} \cup l_{t}^{i} \subset \overline{G_{t-\varepsilon}}$,

for any $t \geq 0$.

Proof of Proposition 1. By Theorem 3 we can choose $N$ sufficiently large so that

$$
a_{i}, b_{i}<\frac{\varepsilon}{3} \text { for all } i \geq N,
$$

and

$$
\left|\rho(x, y)-\rho^{i}(x, y)\right|<\frac{\varepsilon}{3} \text { for all } i \geq N \text { and } x, y \in G^{i} .
$$

Let us take any $x \in G_{t+\varepsilon}$. Then $\rho(x, \partial G)>t+\varepsilon \geq \varepsilon$, and so by the restriction on $b_{i}$ in (9) we must have $x \in G^{i}$ for all $i \geq N$. Now, for $i \geq N$,

$$
t+\varepsilon<\rho(x, \partial G) \leq \rho(x, y) \leq \rho(x, z)+\rho(z, y),
$$

for any $y \in \partial G$ and $z \in \partial G^{i}$. The restriction on $b_{i}$ allows us to choose $y=y(z)$ to give

$$
t+\varepsilon<\rho(x, z)+\frac{\varepsilon}{3}<\rho^{i}(x, z)+\frac{2 \varepsilon}{3},
$$


still for all $z \in \partial G^{i}$, and therefore

$$
\rho^{i}\left(x, \partial G^{i}\right) \geq t+\frac{\varepsilon}{3}>t,
$$

which implies that $x \in G_{t}^{i}$, and completes the first part of the proposition.

For the second part, let us take $x \in G_{t}^{i} \cup l_{t}^{i}$ for any $i \geq N$. Then

$$
t \leq \rho^{i}\left(x, \partial G^{i}\right) \leq \rho(x, y)+\frac{\varepsilon}{3} \leq \rho(x, z)+\rho(z, y)+\frac{\varepsilon}{3},
$$

for all $y \in \partial G^{i}$ and $z \in \partial G$. Choosing $y=y(z)$ with the aid of the restriction on $a_{i}$ in (9), we see that

for all $z \in \partial G$ and hence that

$$
t \leq \rho(x, z)+\frac{2 \varepsilon}{3}
$$

$$
\rho(x, \partial G) \geq t-\frac{2 \varepsilon}{3}>t-\varepsilon
$$

which means that $x \in G_{t-\varepsilon} \subset \overline{G_{t-\varepsilon}}$, thus completing the proof of the second part.

口

Proof of Lemma 3. Since the case $t=0$ is vacuous, we may assume that $t>0$. Let us define $Q_{t}^{\varepsilon}=\left(\overline{G_{t-\varepsilon}}-G_{t+\varepsilon}\right)$. Using Proposition 1 we then see that for any $\varepsilon \in(0, t)$ we have

$$
G_{t} \cup l_{t} \subset \overline{G_{t-\varepsilon}}=G_{t+\varepsilon} \cup Q_{t}^{\varepsilon} \subset G_{t}^{i} \cup Q_{t}^{\varepsilon} \subset\left(G_{t}^{i} \cup l_{t}^{i}\right) \cup Q_{t}^{\varepsilon},
$$

and

$$
G_{t}^{i} \cup l_{t}^{i} \subset \overline{G_{t-\varepsilon}}=G_{t+\varepsilon} \cup Q_{t}^{\varepsilon} \subset\left(G_{t} \cup l_{t}\right) \cup Q_{t}^{\varepsilon},
$$

for sufficiently large $i$. In other words, we see that $G_{t} \cup l_{t}$ and $G_{t}^{i} \cup l_{t}^{i}$ can only differ within $Q_{t}^{\varepsilon}$. Therefore, since $P_{t}=G \backslash\left(G_{t} \cup l_{t}\right)$ (and $P_{t}^{i}=G^{i} \backslash\left(G_{t}^{i} \cup l_{t}^{i}\right)$ ) we see, for fixed $t$ (and for sufficiently large $i$ ) that

$$
\begin{aligned}
\left|\omega^{i}\left(P_{t}^{i}\right)-\omega\left(P_{t}\right)\right| \leq & \left|\omega^{i}\left(G^{i}\right)-\omega(G)\right|+\left|\omega\left(G_{t} \cup l_{t}\right)-\omega^{i}\left(G_{t}^{i} \cup l_{t}^{i}\right)\right| \\
\leq & \left|\omega^{i}\left(G^{i}\right)-\omega(G)\right|+\left|\omega\left(G_{t} \cup l_{t}\right)-\omega^{i}\left(G_{t} \cup l_{t}\right)\right| \\
& +\left|\omega^{i}\left(G_{t} \cup l_{t}\right)-\omega^{i}\left(G_{t}^{i} \cup l_{t}^{i}\right)\right| \\
\leq & \left|\omega^{i}\left(G^{i}\right)-\omega(G)\right|+\left|\omega\left(G_{t} \cup l_{t}\right)-\omega^{i}\left(G_{t} \cup l_{t}\right)\right| \\
& +\Omega^{i}\left(Q_{t}^{\varepsilon}\right) .
\end{aligned}
$$

Using parts (2) and (5) of Theorem 3 (and the fact that both $G_{t} \cup l_{t}$ and $Q_{t}^{\varepsilon}$ are compact) we then see that

$$
\limsup _{i \rightarrow \infty}\left|\omega^{i}\left(P_{t}^{i}\right)-\omega\left(P_{t}\right)\right| \leq \Omega\left(Q_{t}^{\varepsilon}\right) .
$$

It remains to let $\varepsilon$ decrease to zero. In this limit, the area of $Q_{t}^{\varepsilon}$ decreases to zero (note that $l_{t}$ has zero area) and hence $\Omega\left(Q_{t}^{\varepsilon}\right)$ decreases to zero; therefore we find that

$$
\omega^{i}\left(P_{t}^{i}\right) \rightarrow \omega\left(P_{t}\right),
$$

as $i \rightarrow \infty$. The convergence of $f_{i}(t)$ to $f(t)$ proceeds more easily along the same lines. 


\section{The proof of Theorem 1}

We have now prepared enough to prove our main result. The general idea is to proceed as far as we can along the lines of the partial proof given in Sect. 3 but on a polyhedral approximating surface, and then study the convergence in the limit of the approximation.

Proof. Let us take the sequence of polyhedral approximations of the surface $G$ as described in Theorem 3. Using (8) of Lemma 1 and the fact that $f_{i}^{\prime}(t) \geq 0$ for $t \in[0, r] \backslash\left\{t_{j}\right\}$ we see that

$$
\frac{d}{d t}\left(f_{i}^{\prime}(t)^{2}\right) \leq 2 f_{i}^{\prime}(t)\left(-\omega^{i}\left(P_{t}^{i}\right)-\tau^{i}\left(\partial G^{i}\right)\right),
$$

for $t \in[0, r] \backslash\left\{t_{j}\right\}$ (cf. section 3 ). Integrating this over an interval $\left(t_{j}, t_{j+1}\right)$ yields

$$
\begin{aligned}
f_{i}^{\prime}\left(t_{j+1}-0\right)^{2} & -f_{i}^{\prime}\left(t_{j}+0\right)^{2} \\
& \leq-2 \int_{t_{j}}^{t_{j+1}} f_{i}^{\prime}(t) \omega^{i}\left(P_{t}^{i}\right) d t-2\left(f_{i}\left(t_{j+1}\right)-f_{i}\left(t_{j}\right)\right) \tau^{i},
\end{aligned}
$$

where we have adopted the abbreviation $\tau^{i}$ for $\tau^{i}\left(\partial G^{i}\right)$. Appealing once more to Lemma 1 as we take a sum over $j$ we find that

$$
f_{i}^{\prime}(r-0)^{2}-f_{i}^{\prime}(0+0)^{2} \leq-2 \int_{0}^{r} f_{i}^{\prime}(t) \omega^{i}\left(P_{t}^{i}\right) d t-2\left(f_{i}(r)-f_{i}(0)\right) \tau^{i} .
$$

Now, since $f_{i}^{\prime}(r-0)^{2} \geq 0, f_{i}^{\prime}(0+0)=f_{i}^{\prime}(0)=L^{i}, f_{i}(0)=0$ and $f_{i}(r)=A^{i}$ (note that $l_{i}(r)$ has zero area) this yields

$$
2 \tau^{i} A^{i} \leq\left(L^{i}\right)^{2}-2 \int_{0}^{r} f_{i}^{\prime}(t) \omega^{i}\left(P_{t}^{i}\right) d t .
$$

It is this statement which we study in the limit $i \rightarrow \infty$, with a view to obtaining (6). Using Theorem 3 we know that $\tau^{i} \rightarrow \tau(\partial G), A^{i} \rightarrow A$ and $L^{i} \rightarrow L$, and therefore we are reduced to proving that

$$
\limsup _{i \rightarrow \infty}-2 \int_{0}^{r} f_{i}^{\prime}(t) \omega^{i}\left(P_{t}^{i}\right) d t \leq-2 \int_{0}^{A} s K^{*}(s) d s .
$$

Our first step in this direction is to use the convergence of Lemma 3 and the bounds of Lemma 2 to secure the dominated convergence

$$
\int_{0}^{r} f_{i}^{\prime}(t) \omega^{i}\left(P_{t}^{i}\right) d t-\int_{0}^{r} f_{i}^{\prime}(t) \omega\left(P_{t}\right) d t \rightarrow 0,
$$

as $i \rightarrow \infty$. (Note that the integrand here is not defined for $t \in\left\{t_{j}\right\}$, but that the union of $\left\{t_{j}\right\}$ over all $i \in \mathbb{N}$ is a countable, and therefore irrelevant set.) Therefore we are reduced to proving

$$
\limsup _{i \rightarrow \infty}-\int_{0}^{r} f_{i}^{\prime}(t) \omega\left(P_{t}\right) d t \leq-\int_{0}^{A} s K^{*}(s) d s .
$$


Using the property of $K^{*}$ given in (3) we may estimate

$$
\begin{aligned}
-\int_{0}^{r} f_{i}^{\prime}(t) \omega\left(P_{t}\right) d t \leq & -\int_{0}^{r} f_{i}^{\prime}(t)\left(\int_{A-f(t)}^{A} K^{*}(s) d s\right) d t \\
= & -\int_{0}^{r} f_{i}^{\prime}(t)\left(\int_{A-f_{i}(t)}^{A} K^{*}(s) d s\right) d t \\
& -\int_{0}^{r} f_{i}^{\prime}(t)\left(\int_{A-f(t)}^{A-f_{i}(t)} K^{*}(s) d s\right) d t,
\end{aligned}
$$

where we extend the definition of $K^{*}(s)$ to be identically zero for negative $s$ in case $f_{i}(t)>A$ for some values of $i$ and $t$. Rewriting the first term and estimating the second using Lemma 2 (and the boundedness of $\left|K^{*}\right|$ and $A^{i}$ ) we see that

$$
\begin{aligned}
&-\int_{0}^{r} f_{i}^{\prime}(t) \omega\left(P_{t}\right) d t \leq \int_{0}^{r} \frac{d}{d t} \int_{A-f_{i}(t)}^{A}\left(A-f_{i}(t)-s\right) K^{*}(s) d s d t \\
&+C \int_{0}^{r}\left|f(t)-f_{i}(t)\right| d t \\
&= \int_{A-A^{i}}^{A}\left(A-A^{i}-s\right) K^{*}(s) d s+C \int_{0}^{r}\left|f(t)-f_{i}(t)\right| d t \\
& \leq-\int_{0}^{A} s K^{*}(s) d s+C\left(A-A^{i}\right)+C \int_{0}^{r}\left|f(t)-f_{i}(t)\right| d t,
\end{aligned}
$$

for some constant $C$. A final appeal to Theorem 3 (part (3)) and Lemmata 3 and 2 confirms that the final two terms of (13) converge to zero in the limit $i \rightarrow \infty$ which allows us to infer (12).

Acknowledgements. This work was carried out during a year long visit to the ETH in Zürich. The author would like to express his thanks to the Forschungsinstitut für Mathematik and to Michael Struwe for their hospitality.

The author received support from the Royal Society through the European Science Exchange Programme.

\section{References}

[1] Alexandrov, A.D. and Zalgaller, V.A.: Intrinsic Geometry of surfaces. Volume 15, Translations of Mathematical Monographs, Providence, RI: AMS, 1967

[2] Burago, Yu.D. and Zalgaller, V.A.: Geometric Inequalities. Berlin-Heidelberg-New York: Springer, 1988

[3] Fiala, F.: Le problème des isopérimètres sur les surfaces ouvertes à courbure positive. Comm. Math. Helv. 13, 293-346 (1941)

[4] Howards, H., Hutchings, M. and Morgan, F.: The isoperimetric problem on surfaces of revolution of decreasing Gauss curvature. Preprint (1998)

[5] Topping, P.M.: Mean Curvature Flow and Geometric Inequalities. J. reine angew. Math. 503, 47-61 (1998) 